\title{
DISCLAIMER
}

This report was prepared as an account of work sponsored by an agency of the United States Government. Neither the United States Government nor any agency thereof, nor any of their employees, makes any warranty, express or implied, or assumes any legal liability or responsibility for the accuracy, completeness, or usefulness of any information, apparatus, product, or process disclosed, or represents that its use would not infringe privately owned rights. Reference herein to any sfecific commercial product, process, or service by trade name, trademark, manufacturer, or othervise does not necessarily constitute or imply its endorsement, recommendation, or favoring by the United States Governnent or any agency thereof. The views and opinions of authors expressed herein do not necessarily state or reflect those of the United States Government or any agency thereof.

\section{THE STUDY OF MATERIAL PROPERTIES USING CHANNELING RADIATION}

A.H. Pantell, J.O. Kephart, R.K. Klein

Stanford Universitv

CONF-8603114--3

Stanford, CA 94305

H. Park

DE86 014226

AT\&T Bell Laboratories

1247 South Ceder Crest Blvd.,

Allentown, Pennsylvania 18103

B.L. Berman

211 Samson Hall

George Washington University

Washington, DC 20052

S. Datz

Oak Ridge National Laboratories

P.O. Box $X$

Oak Ridge, Tennessee 37830

\section{INTRODUCTION}

A possible application for channeling radiation is for investigating the properties of crystals in which the channeling occurs. In this paper we present some general considerations concerning channeling radiation as a measurement technique, and then we proceed to describe several specific examples.

\section{GENERAL CONSIDERATIONS}

Perhaps the first question to pose regarding any measurement procedure is whether or not the procedure alters the properties under observation. In particular, does the channeling cause a significant temperature rise in the crystal or induce a high density of defects?

Under the conditions for which we performed our measurements, the answer to both these questions is "no". Using an electron beam, the particle energy range was from 10 to $80 \mathrm{MeV}$, at an average current of $\sim 10^{-13}$ A over an area of $0.2 \mathrm{~cm}^{2}$, with an exposure time of - 30 minutes for a channeling radiation spectrum in a $20 \mu$ thick Si crystal, for example, the temperature rise, considering radiation cooling only, is less than $2 \times 10^{-4} \mathrm{~K}$, and the fraction of $\mathrm{Si}$ atoms displaced by the incident electron beam is about 1.5 parts in $10^{12}$. These effects are negligible.

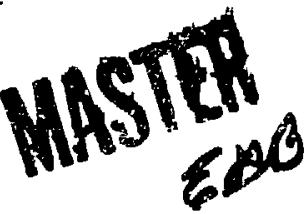


Table I: Some methods for studying crystal properties

\section{Electron Microscopy Ion Channeling X-ray Diffraction Channeling Padiation}

$\begin{array}{lll}\begin{array}{l}\text { High resolution } \\ \text { photography }\end{array} & \begin{array}{l}\text { Location and identifi- } \\ \text { cation of impurity }\end{array} & \begin{array}{l}\text { Measure bulk } \\ \text { phenomena: atomic } \\ \text { atoms. }\end{array} \\ \begin{array}{ll}\text { Spacing, vibrational } \\ \text { Depth imited }\end{array} & \text { Depth limited } & \text { amplitudes } \\ \text { to } \sim 1.0 \mu & \end{array}$

Uses electrons Uses positive to a few MoV energy.

information abtained from the intensity of range from a $F E W$ $\mathrm{KeV}$ to a FEW MeV

\author{
Measure bulk \\ phenomena: \\ crystal potentials. \\ vibrational \\ amplitudes. \\ information \\ obtained from \\ radiation character- \\ istics. Electron or \\ positron energies \\ range from $1.0 \mathrm{MeV}$ \\ to $10 \mathrm{GeV}$.
} a diffracted wave.

$$
\begin{aligned}
& \text { Can be performed } \\
& \text { on thick samples. }
\end{aligned}
$$

\section{Sample thicknesses range from $\mu$ to $\mathrm{mm}$}

can be a problem
Electrons and positrons are different probes.

There are a variety of methods for studying crystal properties. and Table I lists some of these approaches along with their features. These procedures complement each other, in that they can measure different parameters or, in the case that the same parameter is measured, a confirmation is provided. The properties measured by channeling radiation a sinilar to those determined by $x$-ray diffraction in that parameters are averaged over many lattice sites, whereas electron microscopy and ion channeling are sensitive to point defects and impurities.

At low particie energies, sub-MeV to several MeV, an electron microscope or Van de Graaff accelerator could provide the particles; below $30 \mathrm{MeV}$ a single klystron linac or a microtron could be used; and a linac would be required for the higher energies. It is unlikely that a high energy machine would be constructed to channeling radiation studies, tut for an existing accelerator the incremental cost $i: i$ not great.

An interesting feature of channeling radiation as applied to material studies is that electrons and positrons provide different probes. The reason for this is that positrons channel between axes and planes, whereas electrons channel close to axes and planes.

in Table II, the various radiation parameters are listed, along with crystal properties related to these parameters. Our research program, thustar, has utilized photon energy and spectral linewidth and intensity, to obtain information about the crystals. Photon energy. for some range of parameters, can be measured to better than one percent accuracy, whereas linewidth and intensity are determinable to $10-15 \%$ aceuracy. however, it some crystal characteristic is altered, then the change in linewidth and intensity can be obtained within several percent. 
Table II Radiation Parameters and Crystal Properties

Radiation oarameter

Photon energy

Linewidth

Intensity

Time structure

Direction

Polarization

\section{Crystal propenty}

Potential function, vibrational amplitude, electronic bonding.

Vibrational amplitude, multiple scattering. defect density.

Scattering, potential function.

Additional radiation parameters (time siructure, direction, and polarization) might also be used for examining crystals. For exampla, our linac provides hard $x$-rays with about 10 psec duration, which could be applied to a study of ultrafast relaxation processes. Streak cameras, for detection purposes, are sensitive to wavelengths below one Angstrom with a time resolution of 10-20 psec.

\section{i. MEASUREMENT OF CRYSTAL FOTENTIALS}

The location of the emission peaks in the channeling radiation spectra are specified by the crystalline potential function and by the parameters of the incident particle. A procedure that has been applied to the determination of crystalline potential function with undetermined coefficients, and then use a minimization of error technique to match the calculated spectra to the data. In this manner, a potential is obtained which can be cherked against the portion of the data that was not used for the matching.

One example concerns the contribution of the covalent bonding slectrons in diamond to the potential experienced by a channeled electron. 2,3 We usually calculate the potential assuming irdependent free atoms or ions in the crystal, and then summing the individual Hartree-Fock potentials. In Figure 1 the function obtained in this manner, designated as the "free atom" curve, is for electrons channeled by (111) planes in diamond. Another curve, based upon $x$-ray diffraction data, is also shown, and this latter curve incorporates electron bonding whereas the former does not. Eigenvalues are drawn for $30.5 \mathrm{MeV}$ electrons using the x-ray data.

Figure 2 illustrates the spectra calculated from the two curves in Figure 1 for 30.5 and $545 \mathrm{MeV}$ electrons, along with the experimental data. In both iristances the $x$-ray diffraction potential provides a better match to the data. The broad emission peak that occurs at $-30 \mathrm{keV}$ the upper figure and at $\sim 60 \mathrm{keV}$ in the lower results from the addition of $\Delta n=1$ transitions between the eigenstates of Figure 1 . Vibrational motion of the atoms is included in the analysis, both as a Debye-Waller factor and as a line-broadening mechanism. The presence of a $\Delta n=3$ transition, the $3 \rightarrow 0$ for $30.5 \mathrm{NeV}$, is apparent in the data. 


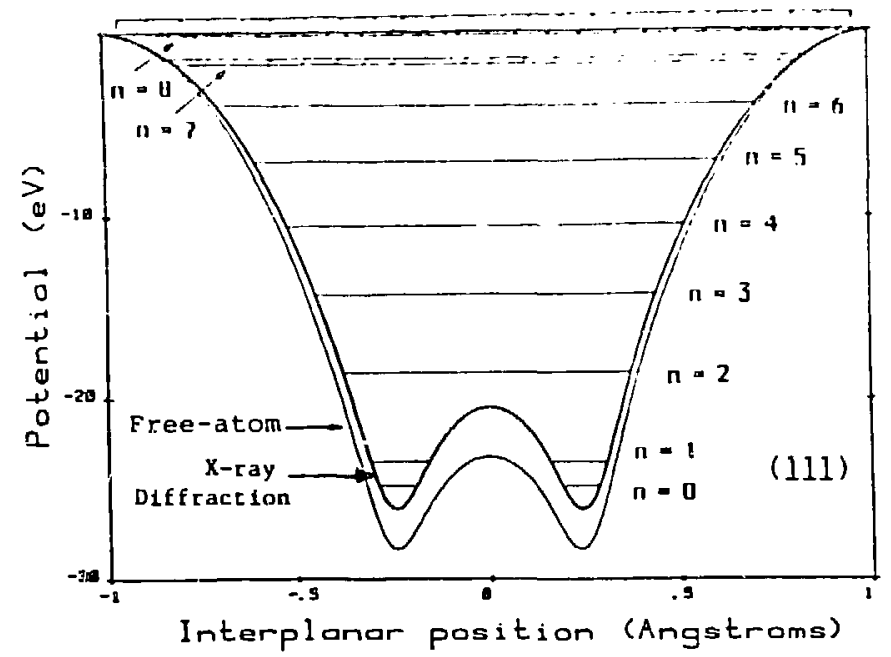

Figure 1: Potential functions for electrons channeling by (111) planes in diamond. $X$-ray diffraction data inciudes valence electron asymmetry, and the free-atom calculation does not.
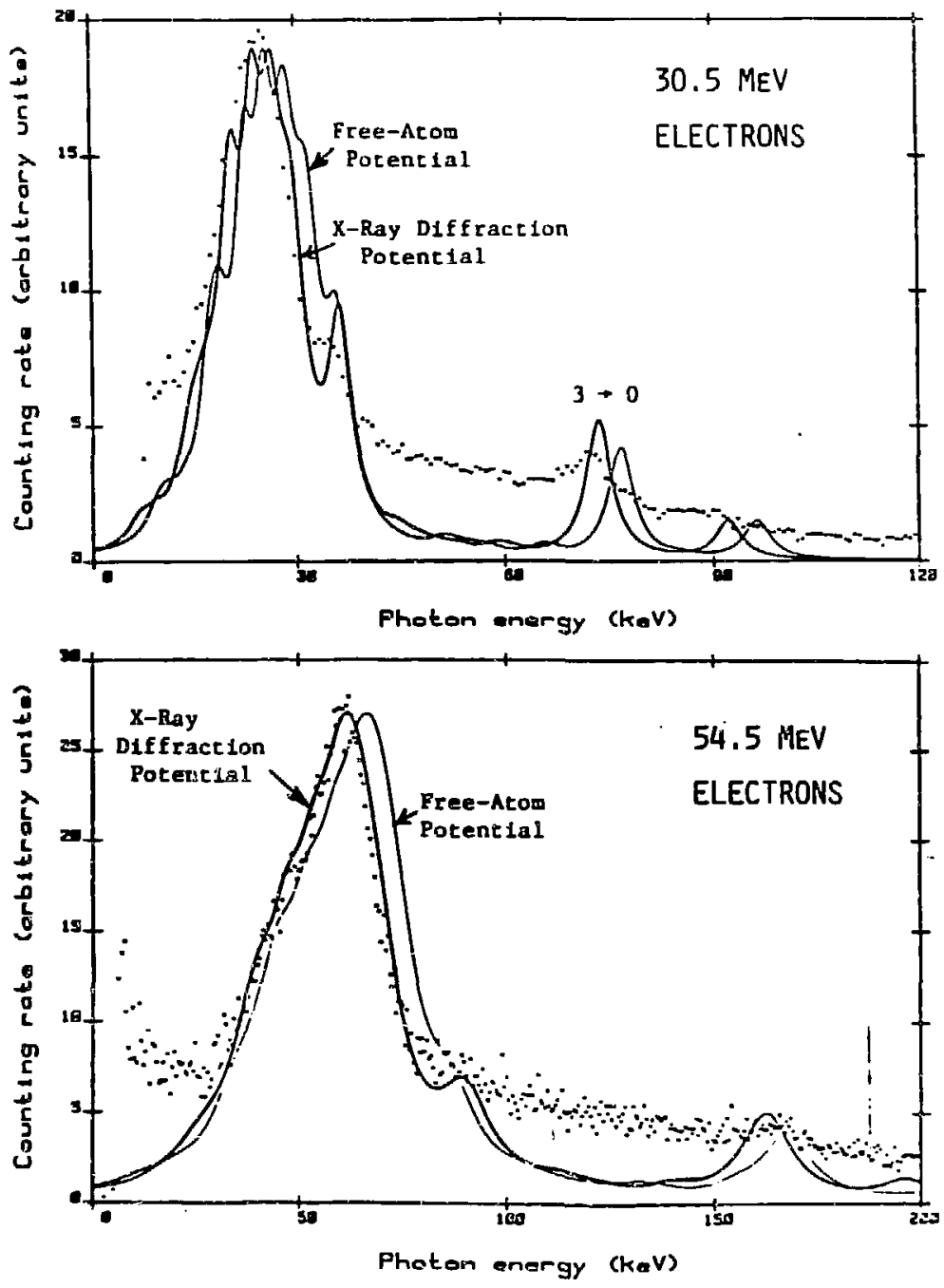

Figure 2: Calculated and Measured spectra from (111) planes. 
With reference to Table III, a new potential was calculated using muasured discrete transitions at different particle energies. In this table a comparison is made between observed ensrgies. and those predicted from the free-atom poiential; the $x$-ray diffracticn potential; and a new polential derived by minimizing the sum of the squares of the percent error for each transition. As seen in Figure 3, this new potential is very similar 10 that derived from $x$-ray diffraction, with a slightly higher potential midway between the choser set of (111) planes.

Table III: Potential calcuation based upon minimization of the sum of (percent error) ${ }^{2}$ for isolated transitions.

\begin{tabular}{|c|c|c|c|c|c|}
\hline \multirow[b]{2}{*}{$\gamma$} & \multicolumn{3}{|c|}{ Observed } & Calculated energy using & \multirow{2}{*}{$\begin{array}{l}\text { Derived } \\
\text { from Spectra }\end{array}$} \\
\hline & Transitioni & Energy & Free-Atom & X-Ray Diffraction & \\
\hline 25.57 & $2 \rightarrow 1$ & 9.87 & $9.85(0.0 \%)$ & $9.62(-2.6 \%)$ & $9.75(-1.2 \%)$ \\
\hline 25.67 & $3 \rightarrow 0$ & 20.6 & $21.90(6.3 \%)$ & $20.61(0 \%)$ & $20.58(-0.1 \%)$ \\
\hline 25.67 & $5 \rightarrow 0$ & 28.90 & $31.73(9.8 \%)$ & $29.48(2.0 \%)$ & $29.28(1.3 \%)$ \\
\hline 34.03 & $2 \rightarrow 1$ & 15.1 & $15.16(0.4 \%)$ & $14.93(-1.1 \%)$ & $15.17(0.5 \%)$ \\
\hline 34.03 & $3 \rightarrow 0$ & 32.5 & $33.55(3.2 \%)$ & $31.75(-2.3 \%)$ & $31.69(-2.5 \%)$ \\
\hline 60.76 & $3 \rightarrow 0$ & 72.1 & $76.86(6.6 \%)$ & $73.59(2.1 \%)$ & $73.37(1.8 \%)$ \\
\hline \multicolumn{3}{|c|}{ Normalized $\Sigma(\% \text { Error })^{2}$} & 7.75 & 1.0 & 0.38 \\
\hline
\end{tabular}

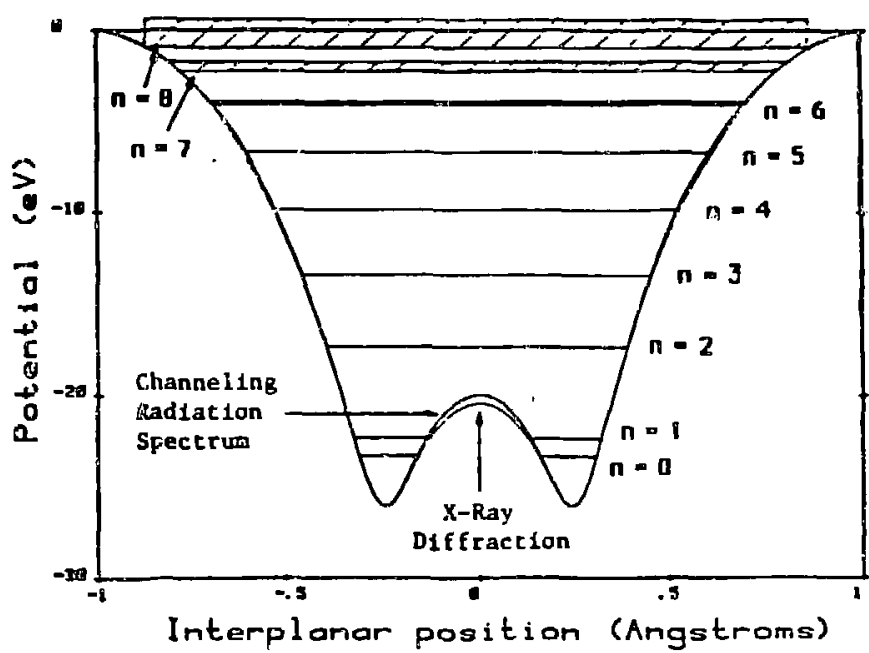

Figure 3: The potantial function derived from the channeling radiation spectrum. 
The final check is to compare theory and experiment for a portion of the data noi used for error minimization. This is done in Fig.4 for the data presented in Fig. 2, and it is seen that the spectrum obtained using the new function from the channeling radiation (C.R.) experiments is essentially indistinguishable from the $x$-ray derived calcuiation, and both have good agreement with the data. This result indicates that channeling radiation spectra may be used to obtain crystalline potential functions with an accuracy comparable to that using $x$-ray diffraction.
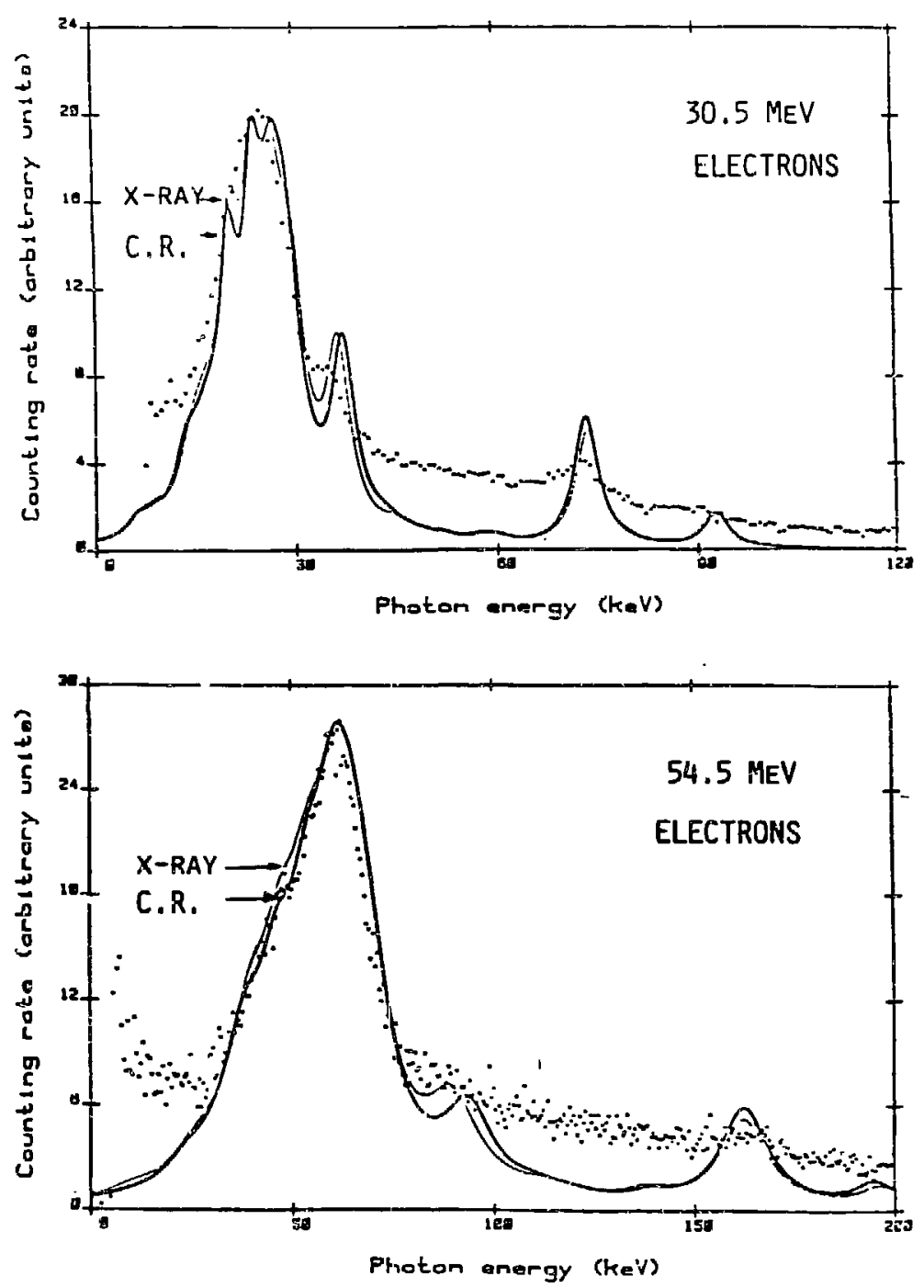

Figure 4: Calculated continuum spectra 


\section{OBSERVATION OF PLATELETS IN DIAMOND}

In certain types of natural diamond (Type la), nitrogen impurities are found in the form of atomic monolayers nucleated on (100) planes. These "platelets" distort the (100) planes, and two proposed models 4,5 for this distortion are illustrated in Fig. 5. A TEM photograph of our Type la diamond sample indicated platelet diameters ranging from 40 to $200 \AA$, with a mean sparation of $\sim 500 \dot{A}$.

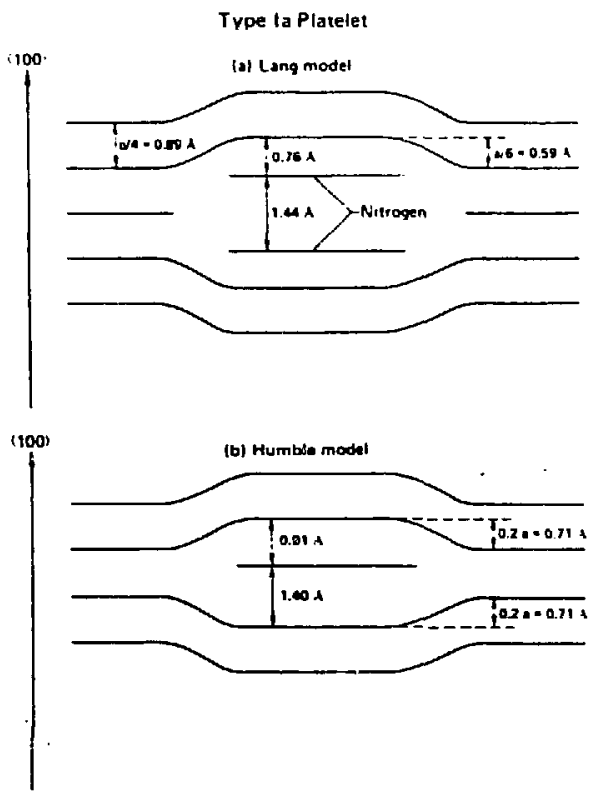

Figure 5: Distortion of $(100)$ planes in diamond due to the presence of nitrogen platelets.

We measured electron channeling radiation from two diamond crystals, one with platelets and one without ( $T$;pe lla), ${ }^{6}$ and the observed photon energies are listed in Tabls IV. Although there was a significant decrease in the intensity of this emission from (1 11 ) planes, there was essentially no change in peak photon energy. This is a reasonable result, since the platelets cause a stacking fault type of defect in (111) planes which increases scattering, but, aside from this discontinuity, the planes r ie undistorted. For the (100) planes, however, there are significant differences in the measured photon energies, with the calculated yalues closer to the measurements obtained for the crystal without platelets. In all our calculations a Debye-Waller factor is introduced to account for atomic vibrations, and for Table IV the published, rms, ong-dimensional amplitude of $0.042 A$ was used. 
Tabie IV: Electron channeling radiation from diamond crystals with and without nitrogen platelets.

\begin{tabular}{lccccc}
\hline Plane & $\begin{array}{l}\text { Electron } \\
\text { Energy }\end{array}$ & Type la & $\begin{array}{c}\text { Photon energy (keV) } \\
\text { Type lla }\end{array}$ & Calculated \\
\hline$(111)$ & $30.5 \mathrm{MeV}$ & $24.2 \pm 0.6$ & $25.0 \pm 0.5$ & 43 \\
$(100)$ & 30.5 & $1 \rightarrow 0$ & $41.3 \pm 0.4$ & $43.0 \pm 0.3$ & 122 \\
$(100)$ & 54.5 & $1 \rightarrow 0$ & $111.0 \pm 2$ & $119.8 \pm 0.7$ & 63 \\
$(100)$ & 54.4 & $2 \rightarrow 1$ & -63 & $64.7 \pm 1.5$ & \\
\hline
\end{tabular}

Figure 6 shows the measured radiation spectra from the two types of diamonds, for $54 \mathrm{MeV}$ electrons channeled by (100) planes. The maximum emission intensity has been normalized to the same value for both curves to emphasize the difference in photon energies. To account for this frequency shift it has been assumed that the platalets introduce an uncertainty in the position of a (100) plane, as illustrated by the sketch shown in Fig. 7. The autocorrelation distance along the plane equals the platelet diameter $(\sim 100 \AA)$, whereas the periodic length of the wiggling motion is $200 \dot{A}$. This suggests that an additional Debye-Waller factor might be used to describe this positional uncertainly, as illustrated by the modified potential in Fig. $\mathbf{B}$.

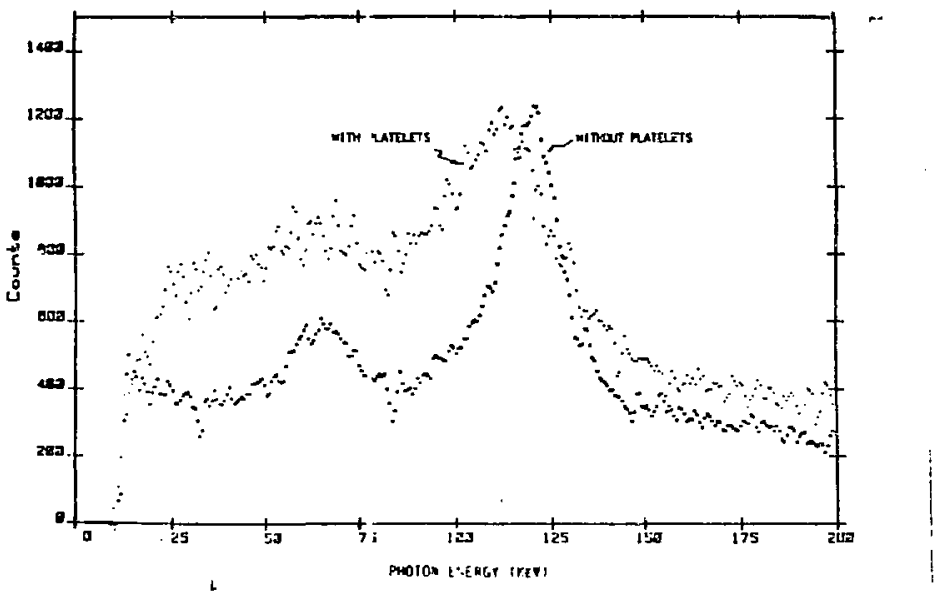

Figure 6: Radiation from $54 \mathrm{MeV}$ electrons channeled along (100) planes. 


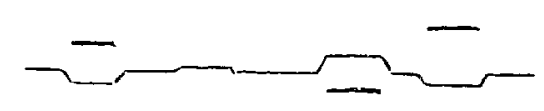

Figure 7: Distortion of (100) planes due to the Platelets

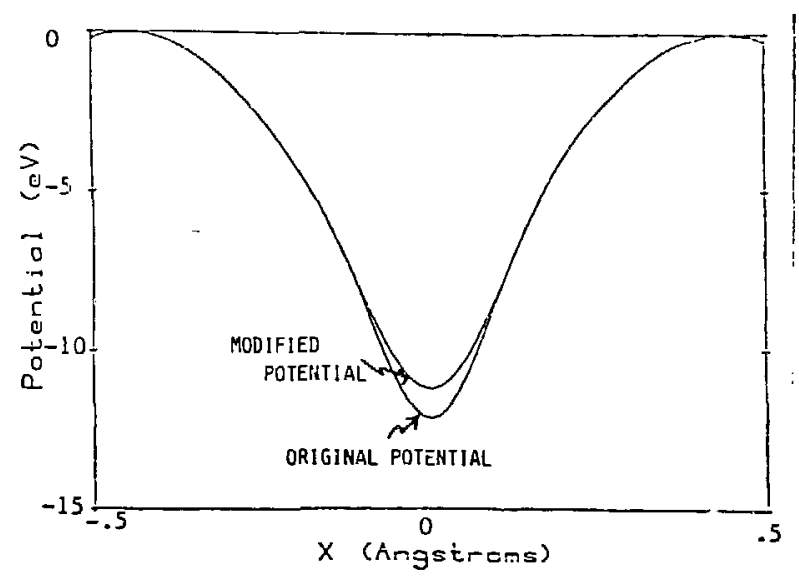

Figure 8 A modified potential function to in corporate the positional uncertainty due to platelets.

Table $V$ contains a tabulation of measured and calculated photon energies for electrons channeled by $(100)$ planes in a diamond with platelets. The calculated values, which are very close to the measured ones, assume a positional uncertainty of $0.038 \AA$ due to platelets, added in quadrature to the $0.042 A$ vibrational amplitude. $A$ value of $0.038 \AA$ is reasonable, 7 from the models in Figure 5 . This result suggests that the effects of the platelets upon the channeled particle may be represent by a Debye-Waller factor, and that the channeling radiation may be used to measure the planar distortion.

Table V: Measured and calculated photon energies from (100) planes in a dlamond crystal with platelets. The calculation uses a positional uncertainty of $\mathbf{0 . 0 3 8} \mathbf{A}$ due to platelets added in quadrature to the $0.042 A$ vibrational amplitude.

Particle Energy Emission Energy for Type la
Calculated Emission Energy from the Modified Potential
$30.5 \mathrm{MeV}$

54.5

54.5
$41.3 \pm 0.4 \mathrm{keV}$

$111 \pm 2.0$
$41 \mathrm{keV}$

113 


\section{DAMAGE EFFECTS IN LiF}

Fifty-four MeV electrons were injected into three LiF crystais, close to the $<100\rangle$ direction, at dosages of $2.5 \times 10^{16}, 2.5 \times 10^{17}$ and $2.5 \times 10^{18}$ electrons per square centimeter. Channeling radiation was then observed from $54 \mathrm{MeV}$ planar-channeled electrons and positrons to determine to effects of the induced damage. Defect inducement is an important consideration for the use of channeling emission as a source of $x$-rays.

Figure 9 shows the channeling radiation spectra from $54 \mathrm{MeV}$ electrons channeled by (110) planes in LiF. Each cystal received a different dosage of electron beam irradiation, as indicated in the figure. Eash spectrum has had the random bremsstrahlung background subtracted. As the dosage increased the spectral peaks became less evident and eventually disappeared in the crystal with the highest dosage. In addition, the linewidths increased as shown in Table 8.
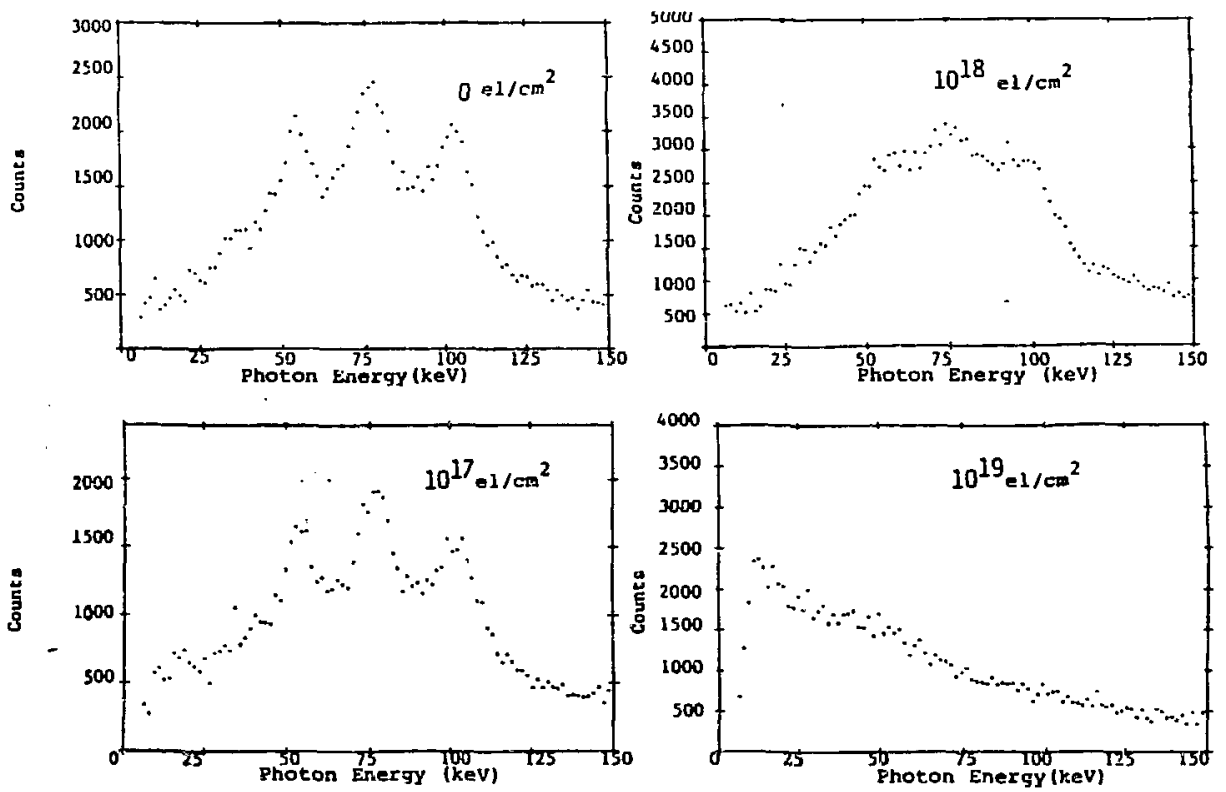

Figure 9: Channeling radiation from $54 \mathrm{MoV}$ eleectron channeled by(110) planes in LiF. The crystals were subjected to defferent dosages of electron beam irradiation: 0 electrons $/ \mathrm{cm}^{2}, 2.5 \times 10^{16}$ electrons $/ \mathrm{cm}^{2}, 2.5 \times 10^{17}$ electrons $/ \mathrm{cm}^{2}$, and $2.5 \times 10^{18}$ electrons $/ \mathrm{cm}^{2}$. 
Table 8: Measured Linewidths for (110) Planar Channeling from 54 MeV Electrons in Damage LiF Crystals

FWHM Measured Linewidths for Different Doses

Transition $h \omega(\mathrm{keV}) \quad 0 \mathrm{el} / \mathrm{cm}^{2} \quad 2.5 \times 10^{16} \mathrm{el} / \mathrm{cm}^{2} 2.5 \times 10^{17} \mathrm{el} / \mathrm{cm}^{2} 2.5 \times 10^{18} \mathrm{el} / \mathrm{cm}^{2}$

\begin{tabular}{|c|c|c|c|c|c|}
\hline $1 \rightarrow 0$ & 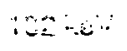 & $\therefore 5$ in' & $;^{-} \ldots$ & $200.0 \%$ & - \\
\hline $2 \rightarrow 1$ & 75 & 14.2 & 15.5 & 17.4 & - \\
\hline $3 \rightarrow 2$ & 54 & 10.3 & 11.6 & 16.1 & - \\
\hline
\end{tabular}

The induced defects are manifested as F-centers, in which the fluorine ion is removed from its site and replaced by an electron. This results in optical absorption, so that an optical absorption spectrum may be used to determine the defect density. Using this procedure, the defect density for the $2.5 \times 10^{17}$ electrons $/ \mathrm{cm}^{2}$ dosage was determined to be $1.5 \times 10^{19} \mathrm{~cm}^{-3}$. This gives a cross section $\sigma$ for $F$-center formation of

$$
\begin{aligned}
\sigma & =\frac{\text { defect density }}{\text { (density of } \mathrm{F} \text { ions) } \times \text { (dosage in electrons } / \mathrm{cm}^{2} \text { ) }} \\
& =950 \mathrm{~b}
\end{aligned}
$$

The tabulated value ${ }^{9}$ is $700 b$, which does not disagree with our measurement since our determination of dosage was not that accurate.

The cross section $\sigma$ for incoherence-inducing scattering of a channeled particle by an F-center may be calculated from the formula

$$
\sigma=\frac{1}{\left(I_{c o h}\right) \times(\text { defect density })}
$$

where $I_{c o h}$ is the coherence length ${ }^{10}$ of a radiating particle. This length may be related to the full-width, half-maximum (FWHM) linewidth $\Delta \omega$ by

$$
I_{\infty}=\frac{\gamma^{2} \lambda}{\pi}\left(\frac{\omega}{\Delta \omega}\right)
$$

where $\lambda$ is wavelength, $\omega$ is frequency, and $\gamma$ is the ratio of the channeled particle energy to its rest energy. Using the linewidths in Table 8 at a dosage of $2.5 \times 10^{17}$ electrons $\mathrm{cm}^{2}$, the value of $\sigma^{\prime}$ is $\sigma^{\prime}=26 \times 10^{16} \mathrm{~cm}^{2}$. At this dosage the coherence 
length due to effects other than the F-centers is relatively small, and has not been included in the calculation.

This value for $\sigma^{\prime}$ is extremely large, considering that the lattice constant is $4.0 \dot{A}$ / The result suggests that the cross section presented by an F-center is comparable to the unit cell area. preliminary results we have obtained in damaged silicon indicate that $\sigma^{\prime}$ is much smaller than in LiF.

Figure 10 shows the channeling radiation obtained from $54 \mathrm{MeV}$ positrons, channeled by $(100)$ planes in the same damaged crystals. It is interesting to note that wnereas there is signiticant degradation in the electron channeling radiaticn at a dosage of $2.5 \times 10^{10}$ electrons $/ \mathrm{cm}^{2}$, there is little change in the shape of the position emission. If one assumes the same defect density and $\sigma^{\prime}$ for positrons as for electrons, the effect of the damage should have been evidenced. Perhaps this means that the defects are localized near the atomic planes rather than midway between.
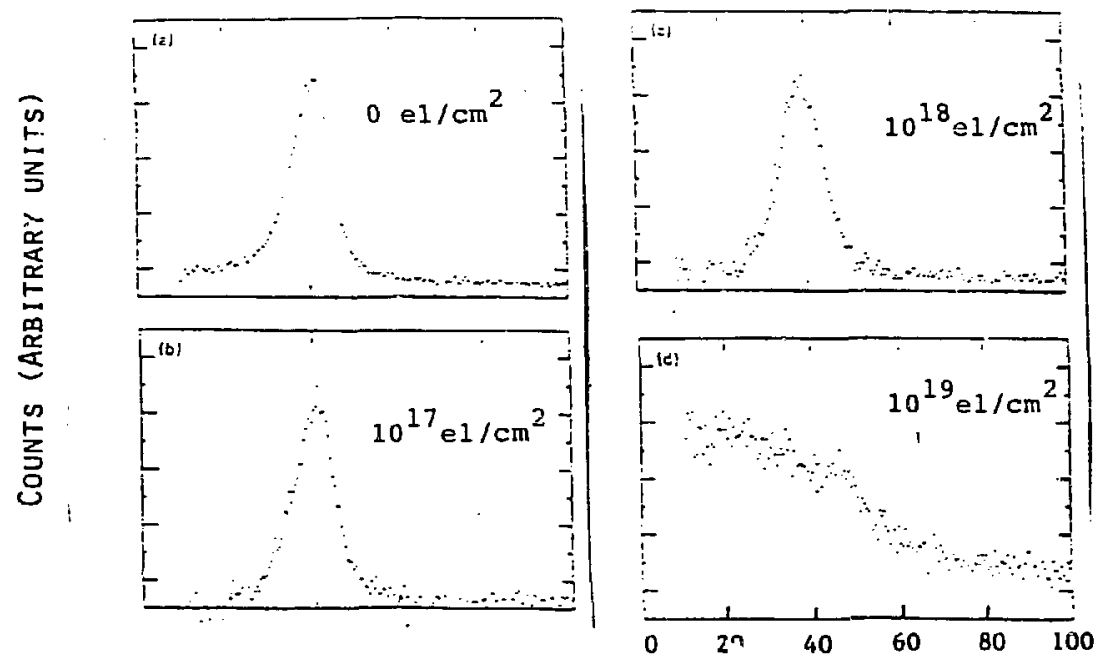

Photon energy (KeV)

Figure 10: Channeling radiation from $54 \mathrm{MeV}$ positrons, channeled by (100) planes in damage LiF crystals.

\section{THERMAL VIBRATION AMPLITUDES}

The vibrational motion of the atoms in a srystal afiects the crystalline potential. Assuming no correlation between the motion of different atoms leads to a Debye-Waller factor ${ }^{10}$, which reduces the depth of the potential in the vicinity of the atomic planes. This effect is shown in Figure 11, where (110) planar potentials for electrons in Si are drawn for crystal temperatures of 80 and 293K. Increasing temperature reduces the well depth and shifts the photon energy of the $1 \rightarrow 0$ transition to lower values. Photon energies for higher transitions are essentially unaffected. 
Si (110) POTENTIAL AT $80 \mathrm{~K}$ AND $293 \mathrm{~K}$

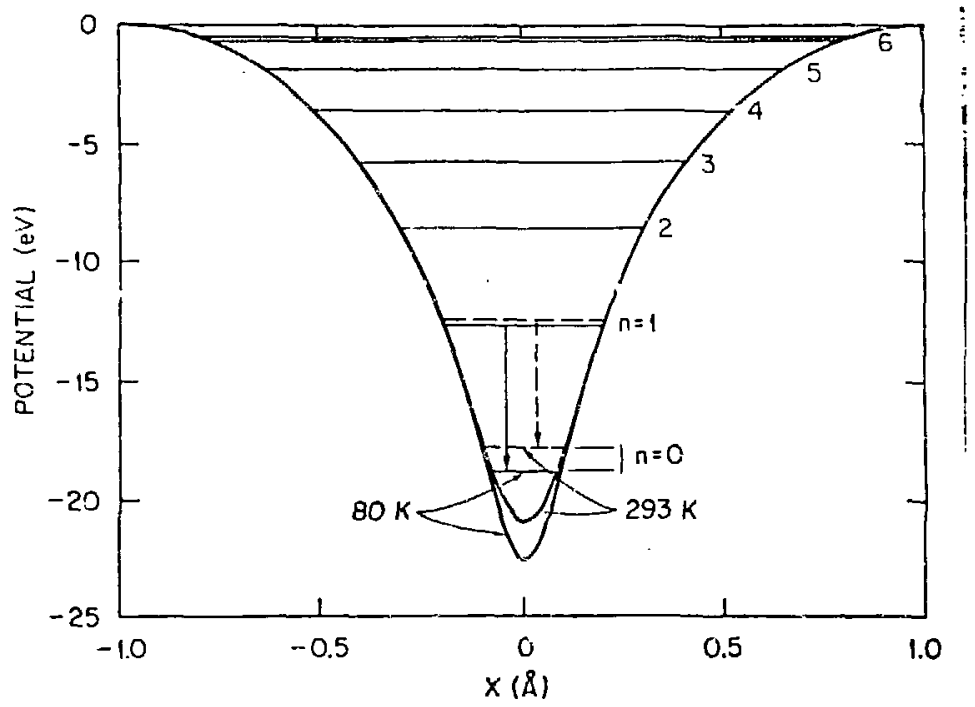

Figure 11: The electron (110) planar potential in $\mathrm{Si}$ at temperatures of 80 and $293 K$ with an assumed Debye temperature of $495 \mathrm{~K}$. Eigenvalues are shown for 51.4 $\mathrm{MeV}$ electrons. 
The observed channeling radiation spectra from $54.4 \mathrm{MeV}$ electrons for two planes in Si are presented in Figure 19 for two different temperatures. As anticipated, at higher temperatures the highest peak photon energy (corresponding to the $1 \rightarrow 0$ transition) in the spectrum is reduced, whereas the photon energies for the other transitions are constant.
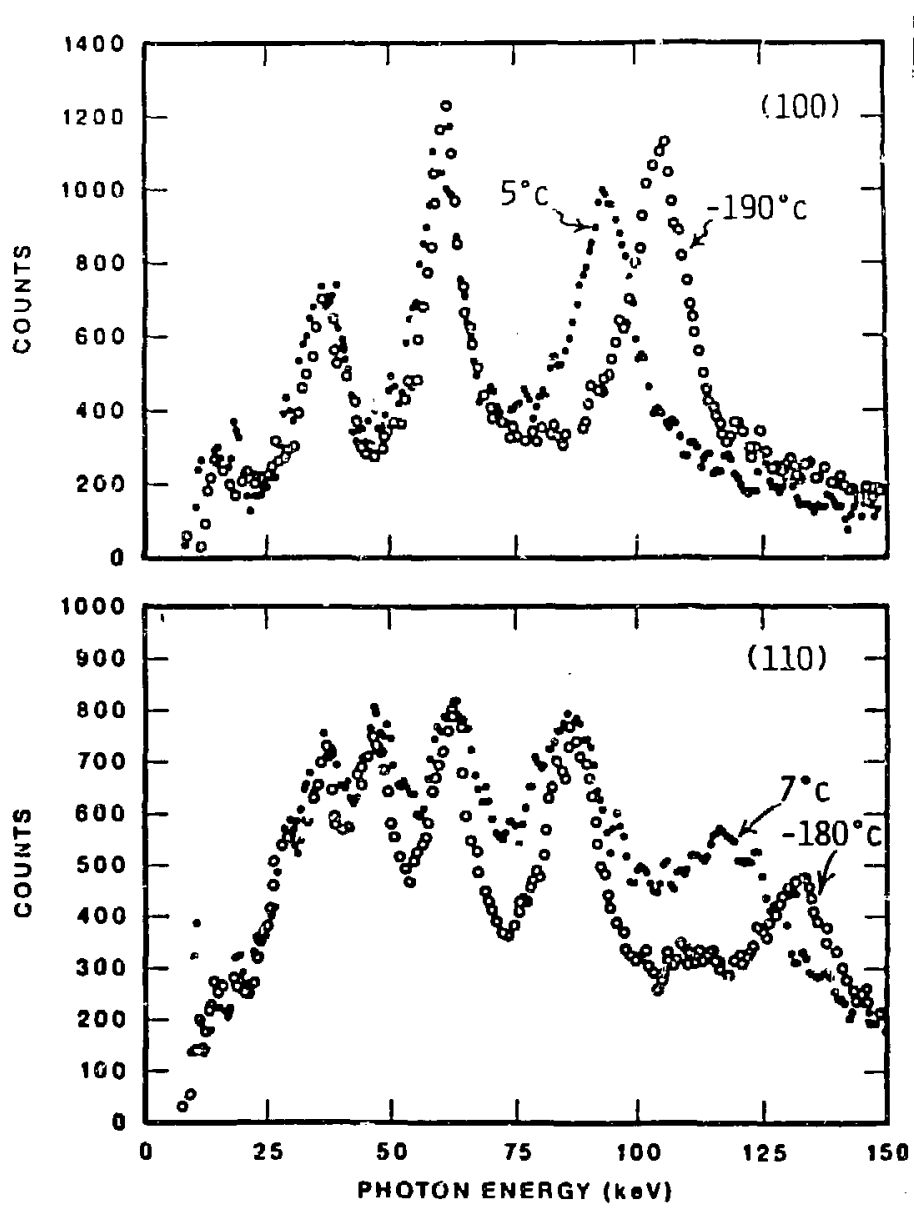

Figure 12: Radiation from $54.4 \mathrm{MeV}$ electrons at different temperatures of the Si crystal. The upper figure is for $\{100)$ planes and the lower is for $(110)$ planes.

As mentioned previously, the depth of the poiential well and thus the $1 \rightarrow 0$ photon energy is a function of the vibrational amplitude, and so the latter can be determined from the radiation spectrum. A surrogate for vibrational amplitude is Debye temperature ${ }^{11}, \theta_{D}$, with a higher $\theta_{D}$ giving a smaller vibration. 
Figure i2 shows the measured photon energies for differant transitions as a function of crystal temperature, using the (110) planes in $\mathrm{Si}$ and $54.4 \mathrm{MeV}$ electrons. The lines drawn through these data points are the calculated energies. For all transitions except $1 \rightarrow 0$, the predicied values are independent of $\theta_{D}$. and the agreement between theory and experiment indicates that we have accurately determined the electron energy, and also that the potential function is vald far region not too close to the atomic planes. For the $1 \rightarrow 0$ transition the predicted photon energies agree with the data using $\theta_{D}=495 K$, whereas the value obtain from $x$-ray diffraction is $\theta_{\mathrm{D}}=549 \mathrm{~K}$.

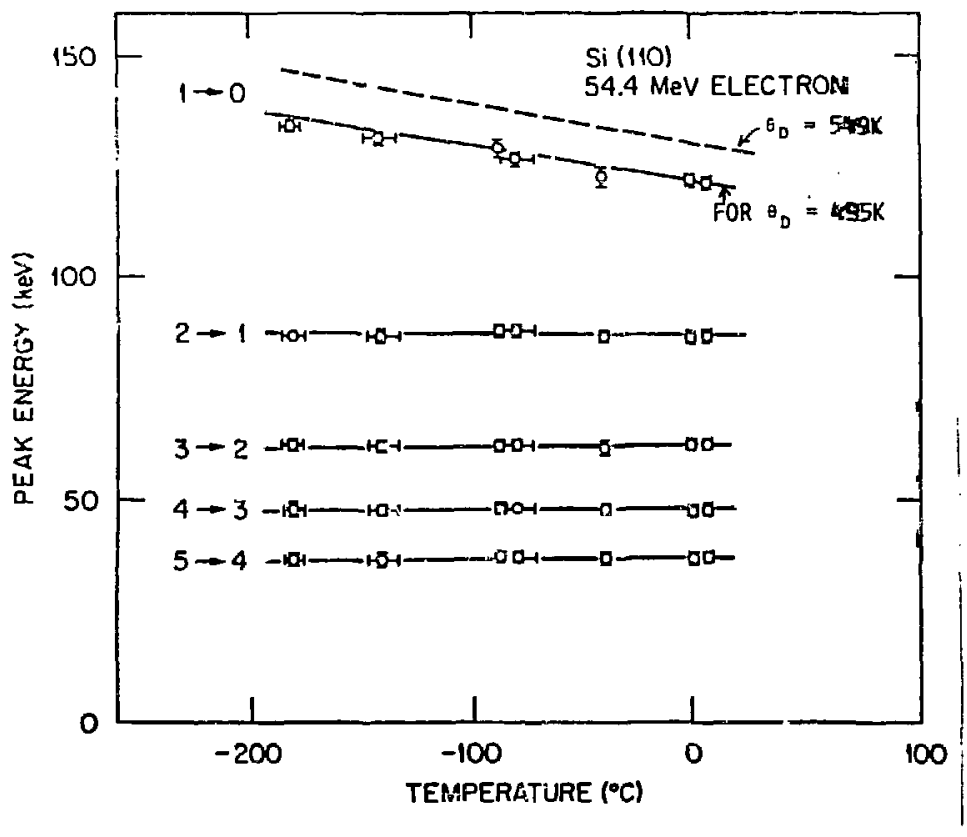

13

Figure 12: Photons energies emitted by $54.4 \mathrm{MeV}$ electrons channieled by (110) planes in Si as a function of crystal temperature.

It is possible that this discrepancy results from the approximation associated with using the Debye-Wailer factor to represent the eflect of thermal vibrations. If this were true, one would expect similar discrepancies for other crystaks, considering crystals with both higher and lower $\theta_{\mathrm{D}}$. Table 9 lists measured and calculated $i \rightarrow 0$ photon energies using the tabulated values for $\theta_{D}$. for four different crystals including Si. From this table it is seen that the largest error occurs for Si and that there is reasonably good agreement for the other three crystals. The reason for the discrepancy that exists in $\mathrm{Si}$ is yet to be determined. 
Table 9: Measured ind calculated photon energies from chanrieling radiation using tabulated values to Debye temperture.

\begin{tabular}{|c|c|c|c|c|c|c|c|c|}
\hline \multirow[b]{3}{*}{2} & \multirow[b]{3}{*}{ Crystal } & \multirow{3}{*}{$\begin{array}{c}\text { Electron } \\
\text { Energy } \\
\text { (Hel) }\end{array}$} & \multirow[b]{3}{*}{$\theta_{D}(K)$} & \multirow[b]{3}{*}{$u(A)$} & \multicolumn{4}{|c|}{$1 \rightarrow 0$ Transition Energy (keV) } \\
\hline & & & & & \multicolumn{2}{|c|}{ (110) plane } & \multicolumn{2}{|c|}{ (100) plane } \\
\hline & & & & & caic. & Meas. & $\bar{C}$ alc. & Meas. \\
\hline 6 & $C($ Diamond $)$ & 54.4 & 2000 & .040 & 163.7 & $161 \pm 0.5$ & 120.6 & $119.8 \pm 0.7$ \\
\hline 14 & si & 54.4 & 543 & .075 & 128.5 & $122.2 \pm 1.0$ & 100.0 & $97.5 \pm 1$ \\
\hline 32 & ge & 16.9 & 290 & .035 & 26.94 & $27.6 \pm 0.5$ & & \\
\hline $31-33$ & GaAs & 16.9 & 260 & .088 & 26.6 & $26.6 \pm 0.4$ & 20.5 & $20.6 \pm 0.5$ \\
\hline
\end{tabular}

\section{CHANNELING RADIATION FROM III-Y GOMPOUNDS, ALLOYS AND SUPERLATTICS}

We have observed planar channeling radiation, usinc $16.9 \mathrm{MeV}$ electrons. from a GaAs crystal, from the alloy $\mathrm{Ga} .7^{\mathrm{Al}}{ }_{3} \mathrm{As}$, and from the superiattice $\mathrm{Ga} .7^{\mathrm{Al}}{ }_{3} \mathrm{As}, / \mathrm{GaAs}$. For the superlattice each layer is $100 \AA$ thick, the intstaces are normal to the $<100>$ direciion, and the channeling for $(100)$ and $(110)$ planes was performed with the direction of relativistic motion normal to the interfaces.

14

Figure $N_{3}$ shows electron planar poteritials in GaAs, $\mathrm{Ga} .7^{\mathrm{Al}} ._{3}^{\mathrm{As} \text {, and }}$ Ga.7Al.3As/GaAs, for the (100), (110) and (111) planes. For the supertattice the potential was calculated by avaraging the concentrations of $\mathrm{Ga}$ and $\mathrm{Al}$ over the layers, so that the potential is equivalent to the alloy $\mathrm{Ga} .85^{\mathrm{Al}} .15^{\mathrm{As}}$.

15

Figure 14 displays the measured and calculated from (110) planes (photons pei electron per steradian per keV vs photon spectra energy) for (a) GaAs, (b) Ga. $7^{\mathrm{Al}} .3^{\mathrm{As}}$,

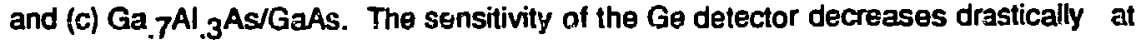
a photon energy of $-10 \mathrm{keV}$, so that data below this energy is not included. Theoretical curves, shown as continuous lines, are based upon an initial $8 \%$ occupation per state (calculated for an electron beam divergence of $1.5 \mathrm{mrad}$ ), and using a different occupation length for each crystal to match the theory with the data. For alf three cases the locations of the spectral peaks agree with the measured values. In Fig. ' $i$ a the occupation length was taken to be $5.5 \mu$, and this gives excellent agreement between theory and experiment. In Fig. ${ }^{4} 4 b$ the occupation length was taken to $2 \mu$, and a length of $3 \mu$ would raise the amplitude of the theoretical curve to match the data for the peak location at $2.5 \mathrm{keV}$. Thus, the occupation length 
curve to match the data for the peak location at $2.5 \mathrm{keV}$. Thus, the occupation length for the alloy is in the range from 2 to $3 \mu$. Similarly, for the superlattice, Fig. 14c, the occupation length is from $1.7-2.4 \mu$.
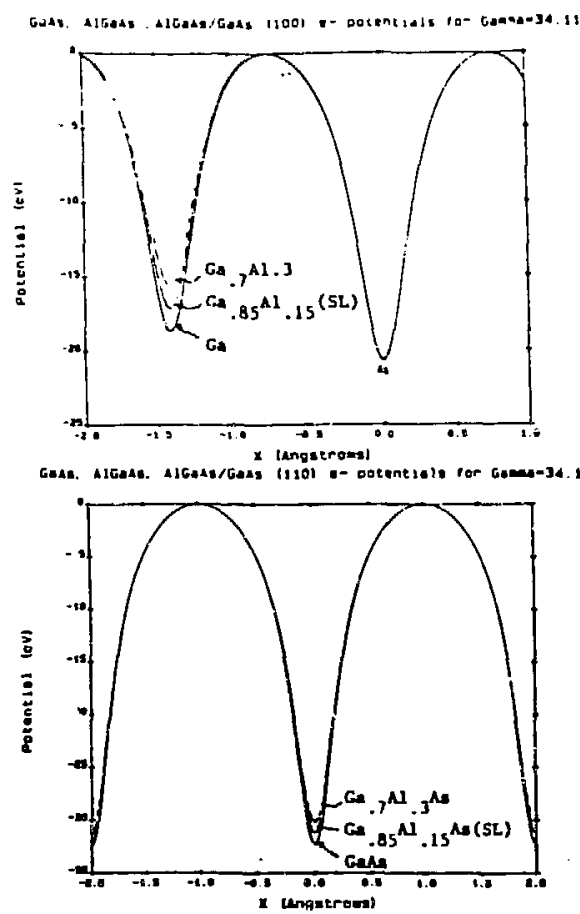

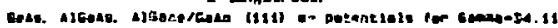

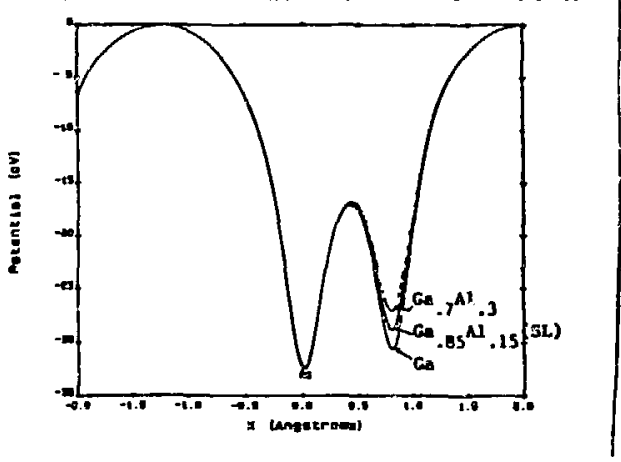

Figure 13: Electron planar potentials for GaAs, Ga. $7^{14} .3^{\text {As }}$ and

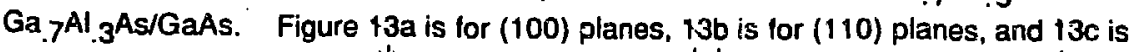
for (111)planes. $14 a$ 


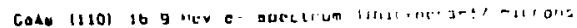

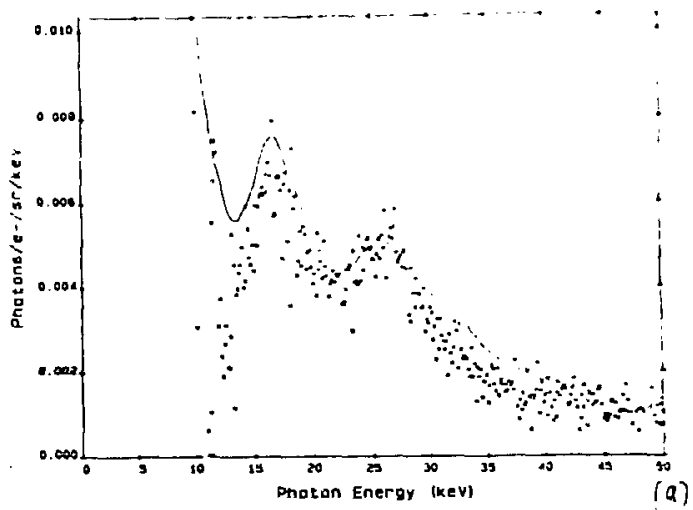

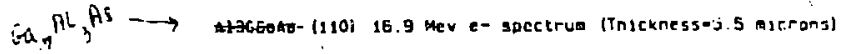

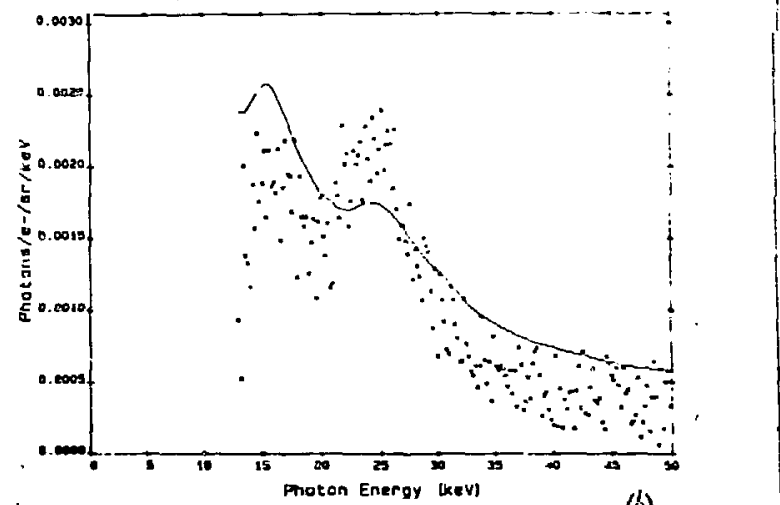

(6)

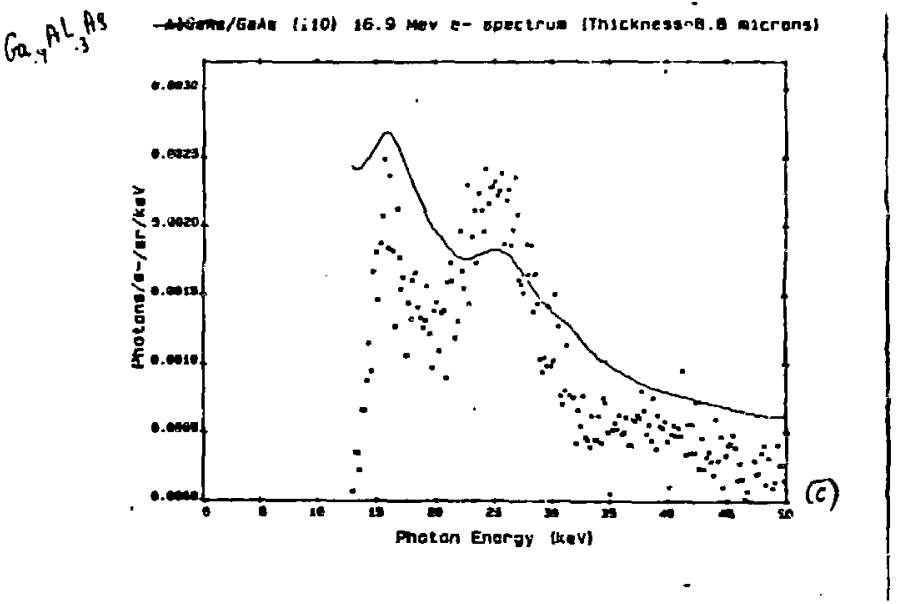

15

Figure 14: Observed and calculated spectra for 16.9 MoV electron channeling

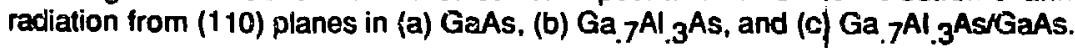

We conclude that the occupation lengths of the channeled electrons in the alloy and superlattice are approximately one-half that in the GaAs. A calculation of the eigenstate mismatch at the interfaces of the superlattice indicates that this is not a significant dechanneling effect. Perhaps there are residual strains or defects in the alloy and sujerlattice that contribute to dechanneling, of perhaps the channeling is disrupted by the random introduction of Al ions. 


\section{OTHER CRYSTAL TYPES AND PROPERTIES TO INVESTIGATE}

An iriterestirig crystal type to investigate by means of channeling radiation is the strained superlattice ${ }^{13}$, (such as $\mathrm{Si}$ : $\mathrm{Ge} / \mathrm{Si}$ ) wherein the lattice constant changes from one layer to the next. Consequence of this is that the normal to a given set of crystal planes may change direction between layers, and so will add another periodicity to the motion of a channeled particle. This results in the generation of new frequencies and sidebands in the emission spectrum, which may be used to identily crystal characteristics.

Other suggested study areas inciude intercalated crystals ${ }^{14}$, in which planes of carbon atoms are separated by a plane of a different atom, and ferroelectrics. wherein the crystal structure and potential change with temperature.

This work was supported by the US AFOSR under Contract No. AFOSR81-0209, and by the US Joint Service Electronics Program under Grant No. DAAG29-85K-0048. The electron positron linac at LLNL received funding from Contract No. W-7405-eng-48, and S. Datz was supported by the US DOE Division of Basic Energy Sciences under Contract No. DE-AC05-840R-21400. The authors also appreciate the assistance provided by Mr. Vikram Krishnamurthy. 